\title{
Manejo de recursos en crisis, importancia de las ayudas cognitivas en anestesia
}

\section{Resource management in crisis, importance of cognitive aids in anesthesia}

\author{
Dr. Luis Leobardo Fortis-Olmedo,* \\ Dra. Natxielhy Fabiola Canseco-Cuevas, ${ }^{*}$ Dr. Rodrigo Rubio-Martínez, ${ }^{\ddagger}$ \\ Dra. María Fernanda Alarcón-Trejo, ${ }^{\S}$ Dr. Daniel Ríos-Gil ${ }^{\S}$
}

\begin{abstract}
Citar como: Fortis-Olmedo LL, Canseco-Cuevas NF, Rubio-Martínez R, Alarcón-Trejo MF, Ríos-Gil D. Manejo de recursos en crisis, importancia de las ayudas cognitivas en anestesia. Rev Mex Anestesiol. 2021; 44 (2): 143-147. https://dx.doi.org/10.35366/99019

RESUMEN. Las ayudas cognitivas son herramientas diseñadas para guiar al usuario en situaciones de crisis, tales ayudas proporcionan instrucciones y garantizan que las tareas a realizar durante la emergencia se lleven a cabo con la mayor exactitud y con esto evitar la omisión de la mayor cantidad de pasos o tareas durante el evento. Está demostrado que las ayudas cognitivas aumentan el rendimiento de los anestesiólogos durante las situaciones de crisis simuladas y más cuando se somete al usuario a casos clínicos raros o de baja incidencia. Presentamos el caso de una mujer de 33 años programada a cirugía electiva, la cual acude súbitamente por probable hipertermia maligna desencadenada por desflurane. Un reader encargado de la lectura de las ayudas cognitivas durante el evento guió las acciones así como el tratamiento sintomático y específico posterior al diagnóstico. Actualmente se recomienda no confiar en la memoria en situaciones de crisis, ya que puede haber omisiones importantes en el diagnóstico, tratamiento y pronóstico.
\end{abstract}

ABSTRACT. Cognitive aids are tools designed to guide the user in crisis situations, such aids provide instructions and ensure that the tasks to be performed during the emergency are carried out with the greatest accuracy and thus avoid the omission of as many steps or tasks during the event. It is demonstrated that cognitive aids increase the performance of anesthesiologists during simulated crisis situations and more when the user is subjected to rare or low incidence clinical cases. We present the case of a 33-year-old woman scheduled for elective surgery, who suddenly presented probable malignant hyperthermia triggered by desflurane. The actions as well as the symptomatic and specific treatment after diagnosis, was guided by a «reader» in charge of reading the cognitive aids during the event. It is currently recommended not to rely on memory in crisis situations as there may be important omissions in diagnosis, treatment and prognosis.

Abreviaturas:

BIS = Índice biespectral.

$\mathrm{CRM}=$ Manejo de recursos en crisis.

EKG = Electrocardiograma.

$\mathrm{ETCO} 2=$ Dióxido de carbono al final de la espiración.

HM = Hipertermia maligna.

PANI = Presión arterial no invasiva.

$\mathrm{TOF}=$ Tren de cuatro.

VMCV = Ventilación mecánica controlada por volumen.

\section{INTRODUCCIÓN}

$E^{\prime}$ l quirófano es un entorno de atención al paciente de alta $\checkmark$ agudeza en el que las crisis, aunque poco frecuentes, ocurren con más frecuencia que en muchos otros entornos de atención al paciente. Las crisis en el quirófano requieren de acciones rápidas y coordinadas en entornos estresantes y críticos por el tiempo, y dependen en gran medida de los conocimientos y habilidades que conservan los médicos. La omisión de los pasos cruciales en el manejo de tales crisis es

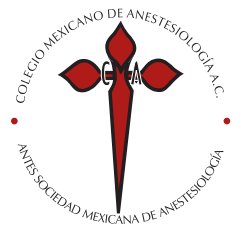

Palabras clave: Ayudas cognitivas, hipertermia maligna.

Keywords:

Cognitive aids, malignant hyperthermia.

\author{
* Anestesiólogo. Centro \\ Médico ABC. \\ ‡ Presidente de la Federación \\ Latinoamericana de Simulación \\ Clínica y Seguridad del Paciente. \\ Coordinador de Anestesia, \\ Centro de Simulación. \\ $\S$ Residente de Anestesiología.
}

Centro Médico ABC. Ciudad de México, México.

Solicitud de sobretiros: Dr. Luis Leobardo Fortis-Olmedo The American British Cowdray Medical Center I.A.P. Sur 136 Núm. 116,

Col. Las Américas, 01120, Alcaldía Álvaro Obregón, Ciudad de México. Teléfono: 52-30-80-00 E-mail: fortis_luis@hotmail.com

Recibido: 06-06-2019 Aceptado: 07-02-2020 común y peligroso para los pacientes ${ }^{(1,2)}$. Existe evidencia que sugiere que el incumplimiento de las mejores prácticas durante las emergencias es común. El interés renovado en el efecto de las listas de verificación para reducir los eventos adversos en la atención médica comenzó a principios de la década de 2000. La evidencia sugiere que las ayudas cognitivas están correlacionadas con un mejor manejo de las crisis de quirófano. Las ayudas cognitivas en la sala de operaciones se basan en la larga experiencia y en la evidencia acumulada de otras industrias de alta confiabilidad, como la aviación y la energía nuclear ${ }^{(1-3)}$. 


\section{CASO CLÍNICO}

Paciente femenino de 33 años de edad, ASA I E, programada para mastopexia con implantes y dermolipectomía, sin antecedentes heredofamiliares de importancia para el plan anestésico, antecedente de anestesia regional para operación cesárea sin incidentes, niega sedaciones o anestesias generales previas. Exploración física con talla: $145 \mathrm{~cm}$, peso: $45 \mathrm{~kg}$, IMC 21.4, sin predictores de vía aérea difícil. Premedicación con palonosetrón $0.075 \mathrm{mg}$ y midazolam 1.5 mg IV. Monitorización: EKG (DII-V5), PANI, pletismografía, pulsioximetria, BIS, capnografía, capnometría, analizador de gases, TOF, espirometría y temperatura esofágica. Inducción endovenosa con fentanyl $150 \mu \mathrm{g}$, propofol $100 \mathrm{mg}$, lidocaína $60 \mathrm{mg}$ y rocuronio $50 \mathrm{mg}$. Se realiza desnitrogenización y con TOF de cero se efectúa laringoscopía directa hoja Macintosh \# 3, Cormack-Lehane I, intubación al primer intento tubo orotraqueal \#7.5 alma de acero sin incidentes. Protección ocular, térmica (sabana térmica y calentador de líquidos) y de salientes óseas.

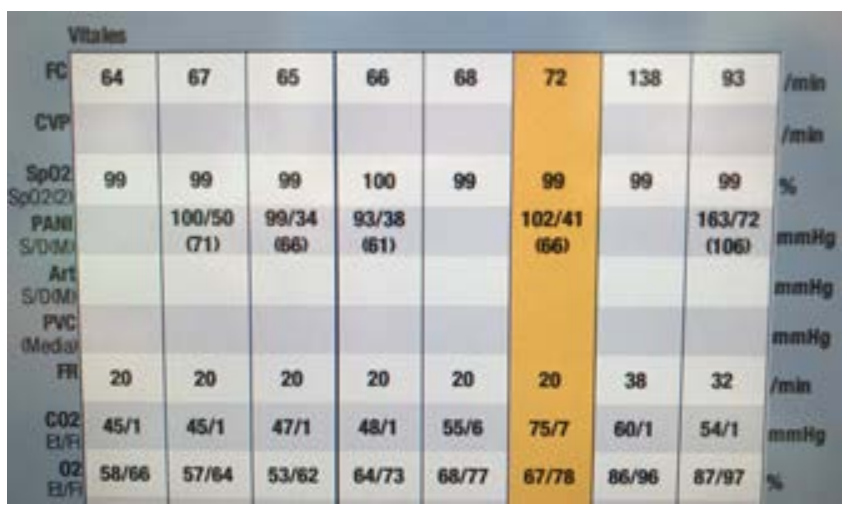

Figura 1: Tendencias de la capnometría: se observa elevación de $\mathrm{ETCO}_{2}$ hasta $75 \mathrm{mmHg}$, con disminución rápida posterior a administración de dantrolene.

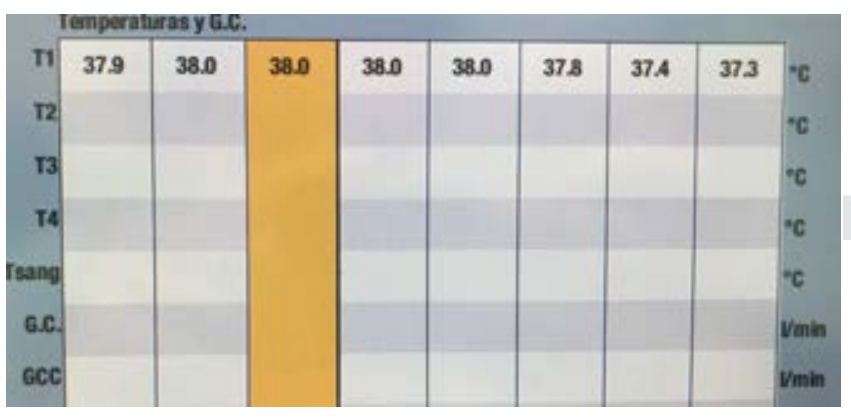

Figura 2: Tendencias de la temperatura esofágica: se observa elevación de la temperatura hasta $38^{\circ} \mathrm{C}$.

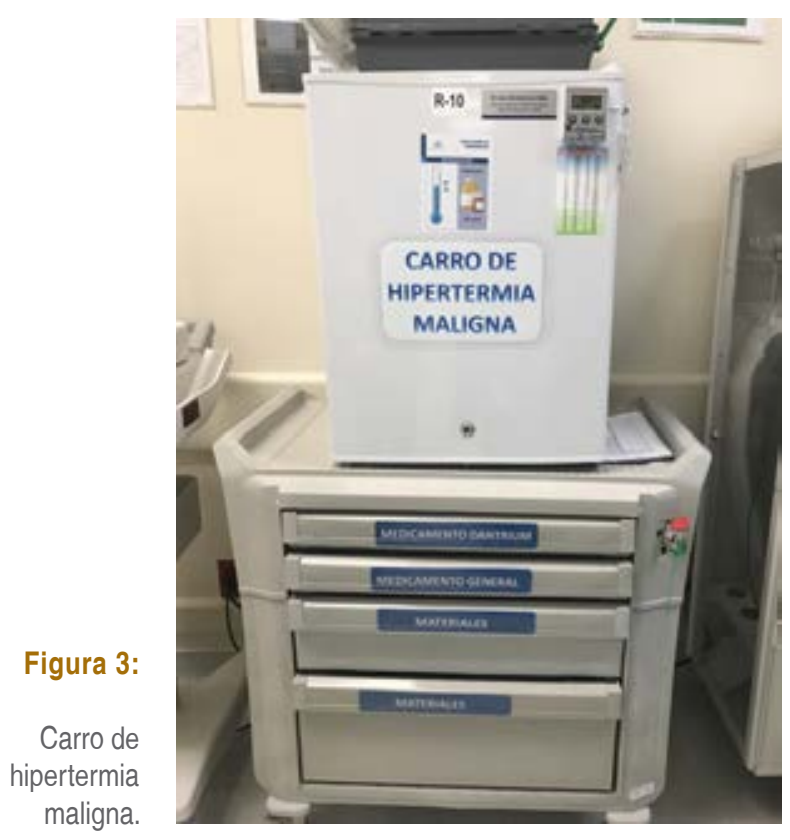

Mantenimiento: VMCV, VC 375 mL, R I:E 1:2, FR 13/ min, flujo de gas fresco $1 \mathrm{~L} / \mathrm{min}, \mathrm{FiO}_{2} 60 \%$, PEEP $6 \mathrm{cmH}_{2} \mathrm{O}$, presión pico 12-15 $\mathrm{cmH}_{2} \mathrm{O}$, desflurane a 6 vol. \%, fentanyl a $0.026 \mu \mathrm{g} / \mathrm{kg} / \mathrm{min}$, dexmedetomidina a $0.3 \mu \mathrm{g} / \mathrm{kg} / \mathrm{h}$ y rocuronio a $10 \mu \mathrm{g} / \mathrm{kg} / \mathrm{min}$, manteniendo BIS 40-60. Adyuvantes: ceftriaxona $1 \mathrm{~g}$, dexametasona $8 \mathrm{mg}$, parecoxib $40 \mathrm{mg}$, ranitidina $50 \mathrm{mg}$.

Transanestésico: se registra aumento de $\mathrm{ETCO}_{2}$ de 28 a 35 mmHg con posterior aumento de la temperatura de 36.1 a 37 ${ }^{\circ} \mathrm{C}$, por lo que se retira soporte térmico. Continúa la elevación progresiva de $\mathrm{ETCO}_{2}$ y temperatura a $75 \mathrm{mmHg}$ y $38^{\circ} \mathrm{C}$ respectivamente (Figuras 1 y 2), con aumento de la frecuencia cardíaca a 138 latidos por minuto, al tener la sospecha del diagnóstico de hipertermia maligna se cierra vaporizador de desflurane, se solicita ayuda, carro de hipertermia maligna (Figura 3) y manual de emergencias (ayuda cognitiva colocado en la máquina de anestesia) (Figuras 4 y 5). Se inicia hipnosis con propofol en infusión a $100-120 \mu \mathrm{g} / \mathrm{kg} / \mathrm{min}$ y se suspende acto quirúrgico. Se establecen roles durante la emergencia y se asigna el rol de reader a una persona, aclarándole que su único papel es leer el algoritmo de hipertermia maligna paso por paso en voz alta y clara.

Se aumenta flujo de gas fresco a $10 \mathrm{~L} / \mathrm{min}$ con $\mathrm{FiO}_{2}$ al $100 \%$ con aumento del VC a $550 \mathrm{~mL}$ y FR 22/min. Primer grupo de ayuda (tres personas) encargado de la toma de gasometría arterial, canalizar otra vía periférica de calibre grueso y enfriamiento con soluciones IV procedentes del refrigerador de hipertermia maligna, lavado vesical y medios físicos. Segundo grupo de ayuda (cuatro personas) enfocado en la preparación del medicamento dantrolene y se administra dosis bolo de 2.5 $\mathrm{mg} / \mathrm{kg}$, dosis total $112 \mathrm{mg}$ (5.6 frascos de dantrolene), dosis única durante evento en quirófano. Se evidencia disminución 


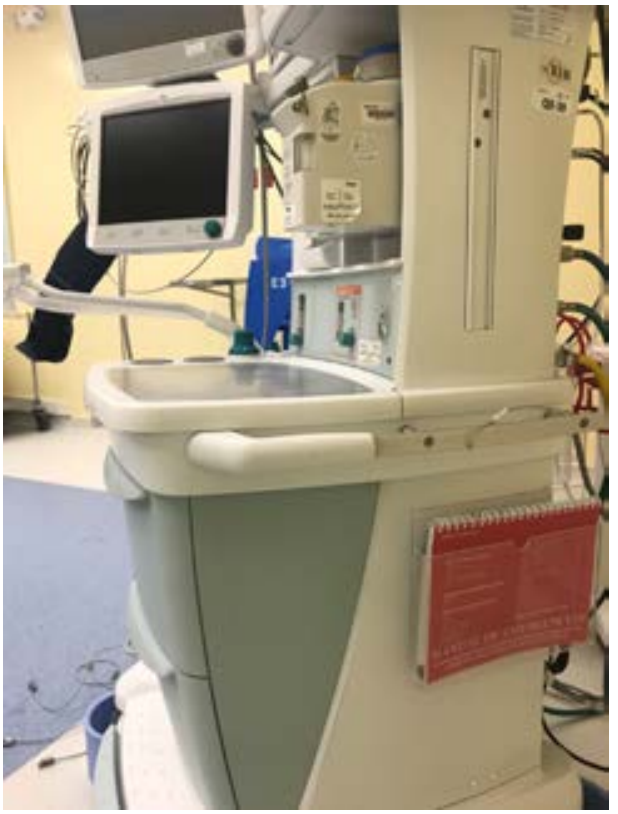

Figura 4:

Localización de fácil acceso del manual de emergencias (a un costado de la máquina de anestesia).

paulatina de los niveles de $\mathrm{ETCO}_{2}$ hasta llegar a $44 \mathrm{mmHg}$ en nueve minutos posterior a dosis bolo y temperatura a $36.9^{\circ} \mathrm{C}$ a los 20 minutos. Acidosis metabólica por gasometría arterial con $\mathrm{pH}$ 7.241, $\mathrm{HCO}_{3} 18 \mathrm{mmol} / \mathrm{L}$ y déficit de base de menos $8.0 \mathrm{mmol} / \mathrm{L}$, por lo que se inició reposición con $\mathrm{HCO}_{3}$. Se avisa al Servicio de Terapia Intensiva y se solicita ventilador, monitor y maletín de traslado.

Ingresa paciente intubada a la UTI, con acidosis metabólica de anión gap bajo e hiperkalemia de 6.8, se inicia apoyo con norepinefrina por TAM $<60 \mathrm{mmHg}$. A las tres horas de su postoperatorio inmediato se realiza corrección ácido-base e hidroelectrolítica con disminución de la sedación, la paciente presenta adecuada interacción al medio, adecuados volúmenes urinarios, sin incremento en la creatinfosfocinasa, afebril y sin datos de rabdomiolisis comprobado por creatinkinasa y lactato deshidrogenasa, por lo que se retira ventilación mecánica, se extuba y se da de alta a piso a las 24 horas. Se explicó a familiar y paciente sobre la probable HM, cuidados posteriores y prueba confirmatoria de biopsia muscular. Sin embargo, al ser una prueba invasiva con un costo de 6,000 a 10,000 dólares efectuada en el extranjero, familiar y paciente se niegan a su realización.

\section{DISCUSIÓN}

La HM es una afección médica muy rara y potencialmente letal con mortalidad reportada de $80-90 \%$ en la década de los 60 , en la actualidad se estima una mortalidad de $5 \%$ gracias a la introducción del dantrolene ${ }^{(4)}$. La HM se presenta como una crisis médica y requiere un tratamiento integral, coordinado e intensivo de recursos. Dado que la incidencia de esta entidad potencialmente mortal es bastante baja, pocos clínicos se preocuparán en realidad por un paciente que experimenta HM durante su entrenamiento como anestesiólogo ${ }^{(5)}$. La supervivencia de una crisis de HM depende en gran medida del reconocimiento temprano y la pronta acción. El empleo de la piedra angular en el tratamiento exitoso de HM, dantroleno, no está disponible en muchas áreas alrededor del mundo debido a su costo, lo que aumenta el riesgo de muertes por $\mathrm{HM}$ en estas zonas ${ }^{(6)}$. El conocimiento médico completo y las habilidades técnicas pueden no ser suficientes para tratar eficazmente a los pacientes que experimentan una emergencia como la HM, ya que este tipo de emergencias son tan raras que es probable que ningún anestesiólogo acumule suficiente experiencia clínica personal en el manejo de cada una para que se le considere un verdadero experto ${ }^{(5)}$.

Las ayudas cognitivas, como las listas de verificación, se han utilizado comúnmente en la aviación desde la década de 1930 y en la energía nuclear, y han demostrado ser herramientas útiles. El término de ayudas cognitivas reconoce que los humanos no están optimizados para recuperar información rara vez utilizada. Las ayudas cognitivas son herramientas creadas para guiar a los usuarios mientras realizan una tarea o grupo de tareas, con el objetivo de reducir errores y omisiones, y aumentar la velocidad y la fluidez del rendimiento ${ }^{(7,8)}$.

Muchas ayudas cognitivas se derivan de documentos que describen secuencias extensas y detalladas de acciones que serían difíciles de examinar al realizar una tarea, particularmente durante una emergencia. Se sugiere que no se debe esperar que los proveedores de atención médica manejen eventos poco comunes sin el uso de una ayuda cognitiva, ya que las listas de verificación de seguridad se han desarrollado y probado con éxito, y utilizado en otras industrias de alto riesgo ${ }^{(7)}$.

Las listas de verificación en un inicio se introdujeron en la anestesia en la década de 1980. En el caso de emergencias clínicas raras como la toxicidad sistémica por anestésicos locales es probable que no sea posible dominar el uso de listas de verificación en un entorno clínico real.

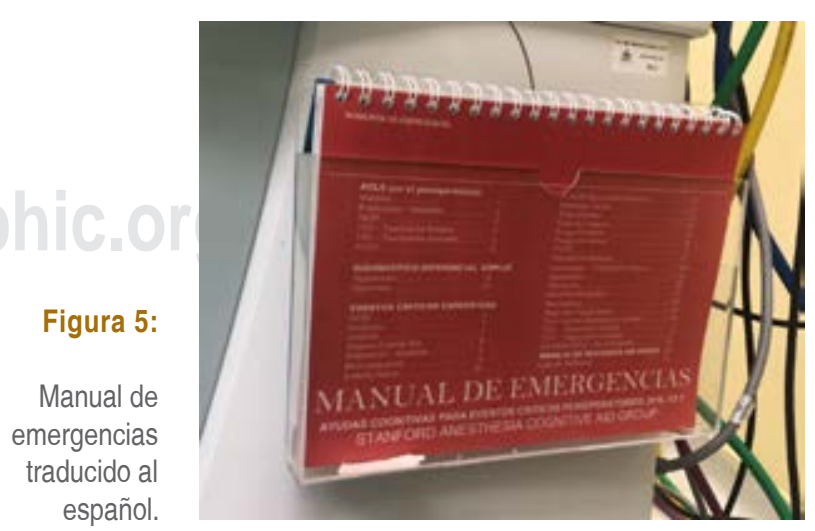


En una situación de crisis surge la pregunta: ¿debería la misma persona que dirige el equipo referirse realmente a la lista de verificación o es más apropiado asignar esta tarea a otra persona? ${ }^{(9)}$

En Virginia se realizó un estudio en el cual se sometió a anestesiólogos a simulación de una emergencia por intoxicación con anestésicos locales, aleatorizados en dos grupos (grupo con lista de verificación y grupo sin lista de verificación). El uso de la lista de verificación mejoró de manera significativa la atención médica y el rendimiento no técnico de los participantes durante un episodio simulado de emergencia grave. El uso parcial de la lista de verificación se correlacionó con un mejor rendimiento general. Además, los participantes con lista de verificación obtuvieron calificaciones más altas en sus comportamientos generales de manejo en situación de cri$\operatorname{sis}^{(10)}$. Otro estudio sobre la simulación de una emergencia de hipertermia maligna demostró que los equipos que obtuvieron los mejores resultados en el tratamiento de la HM utilizaron en gran medida una ayuda cognitiva durante la simulación ${ }^{(11)}$.

Durante la emergencia de nuestro caso asignamos a una persona a buscar el algoritmo de HM para que fuera leyendo paso por paso, ya que la memoria del médico por sí sola puede no ser suficiente para lograr todos los pasos cruciales durante una emergencia ${ }^{(12)}$. Asignar a una persona para leer (reader) la lista de verificación durante una situación de crisis y solicitar los pasos de administración para el equipo mejora la efectividad del uso de la lista de verificación en situaciones de crisis. Eso no es sorprendente, ya que puede ser difícil para el líder de la crisis manejar simultáneamente la situación y hacer referencia a una lista de verificación que está llena de información ${ }^{(9,12)}$.

Asignar un reader puede ayudar a disminuir la carga cognitiva en el líder del equipo durante una crisis. Un área particularmente crucial para el líder y el equipo durante una emergencia es encontrar el equilibrio adecuado entre el diagnóstico, la toma de decisiones y las actividades de distribución de tareas. Esta persona designada como reader permite que el líder y el resto del equipo aprecien mejor la situación ${ }^{(12)}$.

La implementación y el uso del manual de emergencia son dos elementos importantes dentro del contexto más amplio de la capacitación del equipo, así como el manejo de recursos en crisis (CRM).

\section{¿Cuándo y por qué usar manuales de emergencia?}

Existe la idea errónea de que los manuales de emergencia no son relevantes en el manejo de eventos agudos sensibles al tiempo. Ciertamente, puede ser perjudicial consultar un libro o dispositivo computarizado en el momento incorrecto. Entonces ¿por qué no usar tarjetas de bolsillo en lugar de un manual? La respuesta es sencilla debido a que las tarjetas están limitadas por el espacio disponible y es más difícil leer durante una crisis y las tarjetas tendrán fallas en el sistema porque no siempre estarán disponibles ${ }^{(13)}$.

Los manuales de emergencia deben tener un lugar consistente en la sala de operaciones que sea visible y de fácil acceso durante un evento crítico sin bloquear el flujo de trabajo diario $^{(13)}$. En nuestra institución, el manual de emergencias está situado en la parte lateral de la máquina de anestesia, lo que permite su fácil localización por todo el personal de quirófano (Figuras 4 y 5). Aunque también existe el acceso electrónico a los manuales de emergencias, las pruebas de simulación han dejado en claro que las copias físicas son mucho más útiles actualmente durante una crisis ${ }^{(13)}$.

\section{CONCLUSIONES}

La hipertermia maligna es una afección en extremo rara y potencialmente mortal, por lo que una detección y acciones oportunas determinarán el pronóstico del paciente. Al ser una entidad poco frecuente no se debe confiar en la memoria del anestesiólogo para tratar este tipo de crisis ni otras como la intoxicación por anestésicos locales. Para el mejoramiento de los resultados en este tipo de crisis se recomienda contar con ayudas cognitivas situadas en lugares específicos donde todo el personal involucrado en el evento (anestesiólogo, cirujanos, enfermeras, etc.) pueda tener acceso a él. Es importante destacar que tener un manual de emergencia accesible no es una alternativa al aprendizaje y a la enseñanza del material en profundidad. Hay que tener en cuenta también que la preparación para las crisis aún requiere capacitación para lograr la competencia adecuada durante el evento y el uso eficaz de una ayuda cognitiva, esto se puede conseguir a través de la enseñanza en simulación.

\section{SITIO DE INTERÉS}

Grupo de Ayudas Cognitivas en Anestesia de Stanford. Manual de Emergencias: Ayudas cognitivas para eventos críticos perioperatorios 2016, v3.1. Puede descargarse de forma gratuita en el siguiente enlace: http://emergencymanual.stanford.edu 


\section{REFERENCIAS}

1. Alidina S, Goldhaber-fiebert SN, Hannenberg AA, Hepner DL, Singer SJ, Neville BA, et al. Factors associated with the use of cognitive aids in operating room crises : a cross- sectional study of US hospitals and ambulatory surgical centers. Implement Sci. 2018;13:1-12.

2. Ziewacz JE, Arriaga AF, Bader AM, Berry WR, Edmondson L, Wong JM, et al. Crisis checklists for the operating room: development and pilot testing. J Am Coll Surg [Internet]. 2011;213:212-217.e10. Available from: http://dx.doi.org/10.1016/j.jamcollsurg.2011.04.031

3. Hepner DL, Arriaga AF, Cooper JB, Goldhaber-Fiebert SN, Gaba DM, Berry WR, et al. Operating room crisis checklists and emergency manuals. Anesthesiology. 2017;127:384-392.

4. Carrillo-Esper R, Lázaro-Santiago G, Nava-López JA. Hipertermia maligna. Conceptos actuales. Rev Mex Anestesiol. 2013;36:185-192.

5. Scott R, Marjorie I, Stiegler P. Review of crisis resource management (CRM) principles in the setting of intraoperative malignant hyperthermia. J Anesth. 2016;30:298-306.

6. Glahn KPE, Ellis FR, Halsall PJ, Müller CR, Snoeck MMJ, Urwyler A, et al. Recognizing and managing a malignant hyperthermia crisis: guidelines from the European Malignant Hyperthermia Group. Br J Anaesth. 2010;105:417-420.

7. Marshall S. The use of cognitive aids during emergencies in anesthesia: a review of the literature. Anesth Analg. 2013;117:1162-1171.
8. Hoefnagel AL, Rajan S, Martin A, Mahendra V, Knutson AK, Uejima JL, et al. Cognitive aids for the diagnosis and treatment of neuroanesthetic emergencies: Consensus Guidelines on Behalf of the Society for Neuroscience in Anesthesiology and Critical Care (SNACC) Education Committee. J Neurosurg Anesthesiol. 2019;31:7-17.

9. Nanji KC, Cooper JB. It is time to use checklists for anesthesia emergencies simulation is the vehicle for testing and learning. Reg Anesth Pain Med. 2012;37:1-2.

10. Neal JM, Hsiung RL, Mulroy MF, Halpern BB, Dragnich AD, Slee AE. ASRA checklist improves trainee performance during a simulated episode of local anesthetic systemic toxicity. Reg Anesth Pain Med. 2012;37:8-15.

11. Harrison TK, Howard SK, Gaba DM. Use of cognitive aids in a simulated anesthetic crisis. Anesth Analg. 2006;103:551-556.

12. Burden AR, Carr ZJ, Staman GW, Littman JJ, Torjman MC. Does every code need a "reader?" improvement of rare event management with a cognitive aid "reader" during a simulated emergency: a pilot study. Simul Healthc. 2012;7:1-9.

13. Goldhaber-Fiebert SN, Howard SK. Implementing emergency manuals: can cognitive aids help translate best practices for patient care during acute events? Anesth Analg. 2013;117:1149-1161. 